Einführung zum Thema

Anaesthesist 2020 -69:149-150

https://doi.org/10.1007/s00101-020-00728-3

(c) Springer Medizin Verlag GmbH, ein Teil von Springer Nature 2020

Zwei Patienten stellen sich zum Prämedikationsgespräch vor, beide 76 Jahre alt, bei beiden ist ein größerer abdomineller Eingriff geplant. Als Erste betritt eine rüstige Sportlerin das Zimmer. Sie beginnt jeden Tag mit 30 Bahnen im Schwimmbad, lebt zusammen mit Mann, Hund und zwei Katzen, hütet mehrmals wöchentlich ihre Enkelkinder und engagiert sich seit Jahren bei der Tafel ihrer Kirchengemeinde. Der nächste Patient ist ein alleinstehender Rentner, der sich mit Rollator zwar noch in seinen eigenen vier Wänden, jedoch nicht mehr außerhalb der Wohnung bewegt. Sein einziger sozialer Kontakt sind die Mitarbeiter des ambulanten Pflegedienstes, die ihn zweimal täglich bei der Körperpflege und der Bereitstellung seiner Medikamente unterstützen.

Wie diese beiden Fälle verdeutlichen, ist das chronologische Alter eines Menschen hinsichtlich der perioperativen Risikoabschätzung nur wenig aussagekräftig. Inwiefern hilft die ASA-Klassifikation? Sie bietet viel Variabilität innerhalb der einzelnen Klassen, da die Eingruppierung anhand der Ausprägung von „Allgemeinerkrankungen “ erfolgt und Zustände, die nicht per se als Krankheit definiert sind - z. B. Pflegebedürftigkeit im Alter - nicht berücksichtigt werden [6]. Die European Society of Anaesthesiology (ESA) aber auch die US-amerikanischen Gesellschaften für Chirurgie und Geriatrie sowie das (American College of Surgeons [ACS], American Geriatrics Society [AGS]) empfehlen daher, bei der präoperativen Evaluation älterer Patienten spezielle „altersassoziierte“ Risikofaktoren, die sich negativ auf den postoperativen Verlauf auswirken, zu erfassen [1]. Dazu zählen u.a. der funktionelle Status, Multimedikation, inadäquate Medikation, Kognition, Depression, sensori-

\title{
C. Olotu
}

Zentrum für Anästhesiologie und Intensivmedizin, Klinik und Poliklinik für Anästhesiologie, Universitätsklinikum Hamburg-Eppendorf, Hamburg, Deutschland

\section{Wenn nicht Frailty, was dann?}

sche Einschränkungen, Ernährungsstatus, Sturzanamnese und soziale Einsamkeit [3]. Ließe sich die Komplexität des Alterns und ihrer Begleitumstände, die für die sehr individuelle Ausprägung des perioperativen Risikos in dieser Patientengruppe verantwortlich sind, nicht am besten durch solch ein modifiziertes geriatrisches Assessment im Rahmen der präoperativen Evaluation abbilden? Dessen Implementierung in der anästhesiologischen Regelversorgung wird derzeit u. a. dadurch erschwert, dass zwar empfohlen wird, was erhoben werden soll - allerdings nicht wie und womit. Nicht, dass nicht genug gute und validierte Instrumente verfügbar wären, anhand derer sich Depressivität, kognitive Leistungsfähigkeit und funktioneller Status eines Menschen messen ließen. Wer ein solches Assessment in seiner Prämedikationsroutine implementieren möchte, wird jedoch zunächst einige Recherchearbeit aufbringen müssen, um eine geeignete Anwendung zu finden - und dann wahrscheinlich feststellen, dass dieses Instrument ursprünglich nicht mit der Intention entwickelt wurde, das perioperative Risiko eines Patienten abzuschätzen. Bei welchem Testergebnis liegt nun die Grenze, ab der von einem erhöhten Komplikationsrisiko ausgegangen werden kann? Es werden sich nur wenige oder widersprüchliche Angaben zu der perioperativ und klinisch relevanten Ausprägung der einzelnen Risiken finden. Ab wann muss gehandelt werden? Zwar gibt es einige Arbeiten, die sich diesem Thema widmen, die Datenlage ist jedoch derzeit noch überschaubar und wenig konsistent. Die Ermittlung eines klinisch relevanten „Cut-off“-Werts der altersassoziierten Einzelrisiken wird dadurch erschwert, dass häufig mehrere Risiken gleichzeitig bestehen und diese sich, je nach individueller Ausprägung, gegenseitig beeinflussen und potenzieren. Es bleibt demnach zu befürchten, dass auf einen Altersrisiko-Index mit der charmanten Klarheit, wie sie z.B. ein $\mathrm{CHA}_{2} \mathrm{DS}_{2}$-VASc Score zur Abschätzung des Schlaganfallrisikos bei Patienten mit Vorhofflimmern innehat, noch länger gewartet werden muss.

\section{》) In der Risikoevaluation des älteren Patienten hat Frailty das Potenzial zu einem "magic bullet"}

In diesem Zusammenhang kommt der Bestimmung von Frailty, der „Altersgebrechlichkeit“, eine besondere Rolle zu. Frailty umschreibt die Kumulation vieler verschiedener altersassoziierter Risiken wie reduzierte Alltagsaktivität, Mangelernährung, Multimorbidität, funktionelle Abhängigkeit - aber auch kognitive Einschränkungen, Depressivität und soziale Deprivation. Nicht zuletzt aus der Vielzahl der Facetten, die Frailty ausmachen, erklärt sich dessen hohe prognostische Aussagekraft für das perioperative Risiko. Die Erfassung des individuellen Ausmaßes von Frailty hat das Potenzial, sich in der Risikoevaluation des älteren Patienten zu einem „magic bullet“ zu entwickeln, auf das man in der perioperativen Altersmedizin sonst so häufig verzichten muss. Wer „frail“ ist, ist ein Hochrisikopatient - nicht im Hinblick auf ein spezifisches postoperatives Problem, sondern auf nahezu alle erdenklich möglichen Komplikationen.

Dass es auch hier viele Möglichkeiten der Frailty-Bestimmung gibt, aber keine einheitliche Empfehlung, welches Instrument verwendet werden soll, darf nicht 
zur Untätigkeit verleiten. Bislang gibt es keinen Hinweis auf die Überlegenheit einer Methode gegenüber einer anderen im Hinblick auf die individuelle Risikoabschätzung. Bis auf Weiteres gilt also: Es ist nicht entscheidend, wie, sondern dass Frailty präoperativ bestimmt wird. Dies fordert den einzelnen Anästhesieabteilungen ein gewisses $\mathrm{Ma} ß$ an Kreativität $a b$, denn viele der verfügbaren Instrumente zur Bestimmung von Frailty müssen zunächst noch angepasst werden, um in der klinischen Routine praktikabel zu sein. Neben dem Modified Frailty Index [8] verwenden einige Häuser die Handkraftmessung als Indikator für Frailty; an der Berliner Charité kommt ein selbst entwickeltes modifiziertes geriatrisches Assessment zur Anwendung, das sich an der Leitlinienempfehlung der ESA orientiert und damit noch weit über ein FrailtyScreening hinausgeht. In unserer Klinik testen wir den in der Longitudinalen Urbanen Cohorten-Alters-Studie (LUCAS) entwickelten Funktionsindex, einen Patientenfragebogen, der eine Klassifikation von Frailty anhand individueller Risiken und Ressourcen erlaubt [2]. Auf Basis des von Derwall und Coburn vorgestellten Manageable Geriatric (MAGIC) Assessment, entworfen für die Anwendung in der Hausarztpraxis, könnte ein weiteres vielversprechendes Instrument zur präoperativen Bestimmung von Frailty entwickelt werden [4]. Die Vielfalt der Möglichkeiten bietet einen Anreiz für die Initiierung standortübergreifender Forschungsprojekte, um verschiedene Assessmentinstrumente auf die i Machbarkeit ihrer Anwendung hin zu evaluieren und miteinander zu vergleichen.

\section{》) Es ist nicht entscheidend, wie, sondern dass Frailty präoperativ bestimmt wird.}

Welche Methode auch immer benutzt wird, in einem Ergebnis stimmen nahezu alle bislang verfügbaren Studien überein - Frailty ist der Risikofaktor mit der stärksten prädiktiven Aussagekraft für das postoperative Outcome des älteren Patienten [7]. Anders formuliert: Der Verzicht auf ein Frailty-Screening resultiert in einer unzureichenden an- ästhesiologischen Risikoevaluation und führt deren Ziel, „Patienten mit einer bisher nicht erkannten oder nicht ausreichend behandelten und für die Operation oder Anästhesie relevanten Erkrankung präoperativ zu erkennen und die Therapie zu optimieren" [5], ad absurdum.

Ein Screening auf Frailty entbindet nicht von der Leitlinienempfehlung, ebenfalls die kognitive Leistungsfähigkeit, den Ernährungsstatus sowie die weiteren altersassoziierten Risiken präoperativ zu erfassen [3]. Es bietet sich jedoch als erster Schritt im Zuge der Implementierung eines erweiterten präoperativen Assessments für ältere Patienten an.

Der Nachweis von Frailty sollte zu Konsequenzen in der weiteren Versorgung der betroffenen Patienten führen - der Beschreitung ,individualisierte[r] Behandlungspfade“, wie Derwall und Coburn zu Recht fordern [4]. Zeit zur Prähabilitation vor dem elektiven Eingriff, Ernährungssupplementation bei Mangelzuständen und konsequente perioperative Delirprävention können das postoperative Outcome dieser Patienten verbessern. Diese Maßnahmen wirken nur auf den ersten Blick banal, denn hinter ihnen verbirgt sich eine erhebliche Herausforderung für das gesamte Behandlungsteam: In der Regelversorgung sind derzeit weder etablierte Konzepte noch personelle oder finanzielle Strukturen vorhanden, die die Umsetzung eines interdisziplinären perioperativen Behandlungspfades im Sinne eines Multikomponentenprogramms für ältere Hochrisikopatienten erleichtern würden. Die Implementierung dieser Maßnahmen kostet Mühe, Überzeugungsarbeit, Prozessoptimierung, Abkehr von „tradierte[n] Automatismen“ [4] und mitunter auch die Neuregelung von Zuständigkeiten. Dennoch ist es eine Herausforderung, die angenommen werden muss. Dass es gelingen kann, konnte in einigen Anästhesieabteilungen in Deutschland bereits eindrucksvoll demonstriert werden. Wird die Herausforderung gemeistert, geschieht dies nicht nur zugunsten einer Senkung der postoperativen Komplikationsrate im Sinne von Morbidität und Mortalität, sondern v. a. im Hinblick auf den postoperativen Erhalt der Funktionalität, der Selbstständigkeit im Alltag, der kognitiven Leistungsfähigkeit und der Lebensqualität des älteren Patienten; kurzum: im Hinblick auf die Faktoren, an denen nicht nur aus Sicht der älteren Patienten - der Erfolg einer medizinischen Behandlung gemessen werden darf.

\section{Korrespondenzadresse}

\section{Dr. C. Olotu}

Zentrum für Anästhesiologie und Intensivmedizin, Klinik und Poliklinik für Anästhesiologie, Universitätsklinikum Hamburg-Eppendorf Martinistr. 52, 20246 Hamburg, Deutschland c.olotu@uke.de

Interessenkonflikt. C. Olotu gibt an, dass kein Interessenkonflikt besteht.

\section{Literatur}

1. Chow WB, Rosenthal RA, Merkow RP et al (2012) Optimal preoperative assessment of the geriatric surgical patient: abest practices guideline from the American College of Surgeons National Surgical Quality Improvement Program and the American Geriatrics Society. J Am Coll Surg 215:453-466

2. Dapp U, Anders J, Golgert S, von Renteln-Kruse W, Minder CE (2012) Ressourcen und Risiken im Alter. Die LUCAS-I Marker zur Klassifizierung älterer Menschen alsFIT, pre-FRAIL und FRAIL. Validierung und erste Ergebnisse aus der Longitudinalen Urbanen Cohorten-Alters-Studie (LUCAS). Z Gerontol Geriatr 45:262-270.

3. De Hert S, Staender S, Fritsch G et al (2018) Pre-operative evaluation of adults undergoing elective noncardiac surgery: updated guideline from the European Society of Anaesthesiology. Eur J Anaesthesiol 35:407-465

4. Derwall M, Coburn M (2019) Frailty - Ein geeigneter Indikator des perioperativen Risikos alter Patienten? Anaesthesist. https://doi.org/10. 1007/s00101-019-00699-0

5. Deutsche Gesellschaft für Anästhesiologie und Intensivmedizin (DGAI), Deutsche Gesellschaft für Innere Medizin (DGIM), Deutsche Gesellschaft für Chirurgie (DGCH) et al (2017) Praoperative Evaluation erwachsener Patienten vor elektiven, nicht Herz-Thorax-chirurgischen Eingriffen. Anaesthesist 66:442-458 (Gemeinsame Empfehlung der Deutschen Gesellschaft fur Anasthesiologie und Intensivmedizin, der Deutschen Gesellschaft fur Chirurgie und der Deutschen Gesellschaft fur Innere Medizin)

6. Irlbeck T, Zwißler B, Bauer A (2017) ASAKlassifikation. Anaesthesist 66:5-10

7. Mende A, Riegel A-K, Plümer L et al (2019) Gebrechliche ältere Patienten. Dtsch Arztebl Int 116:73-82

8. Subramaniam $S$ et al. (2018). New 5-Factor Modified Frailty Index Using American College of Surgeons NSQIP Data. J Am Coll Surg 226(2):173-181 